\title{
Was Dialektbezeichnungen und Dialektattribuierungen über alltagsweltliche Konzeptualisierungen sprachlicher Heterogenität verraten1
}

\section{Einleitung: Dialektale Kategorienbildung und Dialektbezeichnungen in Wissenschaft und Alltag}

Sprachliche Varianz wird prominent mit Raum in Verbindung gebracht. Sowohl für die Wissenschaft als auch für die Alltagswelt ist die Verknüpfung von Sprache und Raum einer der grundlegenden Ordnungs- und Erklärungsaspekte sprachlicher Variation. ${ }^{2}$

Was die sprachliche Varianz des Deutschen betrifft, die als areal bedingt konzeptualisiert wird, so wird diese in der Linguistik gemeinhin als Kontinuum modelliert. Dieses trägt dem Sachverhalt Rechnung, dass lokale Basisdialekte sich mit zunehmender örtlicher Distanz auch zunehmend voneinander unterscheiden, dass sich aber eine Art von ununterbrochener Verstehbarkeitskette konstituiert (Chambers \& Trudgill 1980: 6). Die zentrale Frage, ob es wissenschaftlich haltbar ist, dieses Kontinuum in Dialekteinheiten zu gliedern, wird seit den Anfängen der Sprachgeographie immer wieder zu einem Zankapfel (zum Problem der Abgrenzbarkeit und Einteilung dialektaler Einheiten vgl. Niebaum 1984, Löffler 2003). In der germanistischen Sprachwissenschaft hat das Bedürfnis nach Kategorienbildung, nach Dialekteinteilungen, die gewissen Ähnlichkeiten und Verschiedenheiten zwischen sprachlichen Ausprägungen Rechnung tragen sollen, seit Jacob Grimm Tradition. Solche Kategorisierungen werden in der Dialektologie meist auf der Basis von Grössen gemacht, die für sprachhistorisch relevant gehalten werden. So sind Niederdeutsch, Mitteldeutsch, Oberdeutsch Beispiele für Kategorien, die in einem definierten Zusammenhang mit dem Phänomen der Hochdeutschen Lautverschiebung stehen.

Auch Dialekteinteilungen von der Art, wie sie von Ferdinand Wrede (1956) vorgenommen wurden, beruhen auf einem Merkmalskatalog: Mit binären Ja-Nein-Entscheidungen - wie wir sie als Prinzip von Linnés Pflanzenbestimmungen her kennen - kommt Wrede beispielsweise zu einer Dialektkategorie, die sich durch die Merkmalsausprägungen ich-Apfel-euch-fescht-Chind auszeichnet. Die Kategorien, die mit diesem Verfahren konstituiert werden, werden jedoch nicht eigentlich begründet. Die Variablen, die den Entscheidungsbaum konstituieren, führen vielmehr zu Dialekträumen, die vorgängig schon etabliert sind; der Entscheidungsbaum macht quasi wissenschaftlich auffindbar, was ethnolinguistisch von alters her als Schwäbisch, Fränkisch, Baierisch usw. bekannt ist.

Stammesnamen wie Schwäbisch, Alemannisch ${ }^{3}$ oder Kantonsbezeichnungen wie Zürichdeutsch ${ }^{4}$ sind bis heute auch in der wissenschaftlichen Literatur durchaus üblich. Mögen diese Bezeichnungen in einigen Fällen blosse Behelfslösungen sein und sich als eine Art von identifizierenden Etiketten auf linguistisch wohl definierte Grössen beziehen (wie etwa auch bei Wiesingers [1983] Einteilung der deutschen Dialekte nach Vokalsystemen), so bezeichnen sie gleichzeitig auch Konzepte anderer, nicht-wissenschaftlicher Natur. Es ist nun aber keineswegs so, dass sich in der Dialektologie die entsprechenden Bezeichnungen immer auf wissenschaftlich definierte Kategorien beziehen würden. Vielmehr scheinen Dialektologinnen und Dialektologen

\footnotetext{
1 Für hilfreiche Kommentare bedanke ich mich bei Evelyn Ziegler (Duisburg/Essen).

2 Zum volkslinguistische Umgang mit Sprache und dessen wissenschaftlichen Aufdeckungsprozeduren vgl. Niedzielski \& Preston (2003).

3 Seidelmann, Erich (2007): Schwäbisch [Hervorhebung H.C.] und Alemannisch am Bodensee. Regensburg.

4 Watts, Richard James: Lokative Präpositionen im Deutschen, Englischen und Zürichdeutschen [Heraushebung H.C.] Eine generativ-transformationelle Analyse. Bern 1976.
} 
oftmals von den alltagsweltlichen Kategorien auszugehen, über die sie genauso wie die Laien verfügen, um dann deren linguistische Eigenschaften zu beschreiben. Auch der Begründer der wissenschaftlichen Dialektologie des Schweizerdeutschen, Franz Joseph Stalder (1819), setzt für seine Bestandesaufnahme der dialektalen Varianz mit einer unhinterfragten Selbstverständlichkeit die Existenz von Kantonsmundarten voraus, die er metonymisch über ortskundige Honoratioren wie Pfarrherren oder Magistratspersonen erhebt. Deren Übersetzung des Gleichnisses vom verlorenen Sohne - insgesamt 38 an der Zahl - verschaffen Stalder die Gewissheit, auf diese Weise Proben ,in allen Schweizermundarten“ (vgl. Stalders Titelei) vorlegen zu können. 5

Die Einleitungen von aktuelleren Dialektgrammatiken und -wörterbüchern (wie z. B. der zürichdeutschen Grammatik (Weber 1987), des Baselbieter Wörterbuchs (Muster \& Bürkli Flaig 2001) oder des bairischen Dialektbuchs (Zehetner 1985), machen deutlich, dass auch hier von Alltagskategorien ausgegangen wird. Da die abgehandelten Dialekte offensichtlich nicht durch ein bestimmtes Set von Merkmalen eindeutig bestimmt sind, wie man dies von einer klassischen wissenschaftlichen Kategorisierung erwarten könnte, sehen sich die Verfasser/innen zu einer Präzisierung des Untersuchungsgegenstandes verpflichtet. So schreibt Weber (1987: 24) präzisierend zum Zürichdeutschen:

\begin{abstract}
Ein ganz einheitliches Zürichdeutsch gibt es nicht: Weder grenzt es sich überall scharf und eindeutig gegen die Mundarten der Nachbarkantone ab, noch ist es in sich überall völlig gleich. Es lassen sich [...] eine nördliche und südliche Gruppe unterscheiden und innerhalb dieser wieder Untergruppen unterscheiden, wobei man diese Einteilung aber nicht pressen darf: Der Verschiedenheiten sind noch viel mehr; wir versuchen eine gewisse Gliederung herauszumodellieren, lassen dabei aber vieles unter den Tisch fallen. [...] Der Verfasser musste, wollte er ein übersichtliches Buch zustande bringen, eine bestimmte Mundart eines engeren Gebietes darstellen und die wichtigsten Abweichungen der übrigen Spielarten des Züritüütsch nur am Rande erwähnen (häufig in Fussnoten), wobei Vollständigkeit unmöglich ist. Er hat die Mundart seiner Heimatgegend gewählt.
\end{abstract}

Das Zürichdeutsche, wie es Albert Weber hier beschreibt, kann nicht einer wissenschaftlichen Kategorisierung entsprungen sein, sondern stellt sich vielmehr als Kategorie mit den charakteristischen Eigenschaften einer natürlichen Kategorie heraus, nämlich: Der Kategorie Zürichdeutsch wird trotz interner Heterogenität eine gewisse Einheitlichkeit nicht abgesprochen; Zürichdeutsch kann metonymisch durch einen Idiolekt vertreten werden; Zürichdeutsch hat nach aussen keine klare Grenze. 6

Dass Weber überhaupt von einer Grösse Zürichdeutsch ausgehen kann und zwar ihre Erscheinungsform, nicht jedoch ihre Existenz an sich zu problematisieren braucht, zeugt davon, dass Zürichdeutsch ein Beispiel für eine ethnolinguistisch relevante Kategorie ist, die ihrerseits offenkundig macht, dass in der Alltagswelt der Wirklichkeitsausschnitt Sprache ebenso in Kategorien gegliedert wird wie dies beispielsweise bei Pflanzen, Tieren, Möbelstücken oder meteorologischen Erscheinungen der Fall ist.

Wie ein Wirklichkeitsausschnitt in welche und in wie viele solcher natürlichen Kategorien aufgeteilt wird, dürfte im Falle der Dialekte mit verschiedenen Faktoren zusammenhängen, mit der auditiven Wahrnehmungsfähigkeit des menschlichen Ohrs und Gehirns, mit der kulturellen Bedeutung sprachlicher Unterschiede und ihrem sozialsymbolischen Wert, mit der individuellen

5 Stalder dokumentiert die Kantonsmundarten mit einer jeweils unterschiedlichen Zahl von Übersetzungen, die unterschiedlichen Gegenden zugewiesen werden; für die meisten Kantone findet sich ein Belegtext, für Bern jedoch deren sechs. Es fragt sich, ob die unterschiedliche Zahl sprachlichen Phänomenen geschuldet ist oder der Zufälligkeit des Bekanntenkreises des Entlebucher Pfarrers Franz Joseph Stalder.

6 Zur alltagsweltlichen Kategorisierung und natürlichen Kategorien vgl. Rosch (1978), Rosch u. a. (1976). Zu einem Überblick von Eigenschaften natürlicher Kategorien vgl. Lakoff (1987: 12f.). 
Zugänglichkeit zu sprachlicher Variation. Eine Antwort auf die Fragen, über welche und über wie viele dialektale Kategorien einzelne Individuen verfügen und welche sprachlichen Ausprägungen welchen dieser Kategorien zugeordnet werden, würde nach einem speziellen psychologischen Testdesign verlangen, das hier nicht konzipiert wurde (vgl. zu Dialektvisualisierungen Berthele 2006). ${ }^{7}$

\section{Alltagsweltliche Dialektbezeichnungen}

\subsection{Dialektbezeichnungen und bevorzugte Kategorisierungsniveaus}

Was im Folgenden in den Blick genommen wird, sind ausschliesslich Kategorienbezeichnungen für Dialekte, wie sie im Deutschschweizer Alltag verwendet werden. Diese Kategorienbezeichnungen dienen als Hinweis daraufhin, welche aussersprachlichen Grössen bei der ethnolinguistischen Kategorienbildung von Relevanz sind (beispielsweise die politische Verwaltungseinheit Kanton bei der Kategorienbezeichnung Zürichdeutsch, eine Ortschaft bei der Kategorienbezeichnung Winterthurer Dialekt) und in einer Sprechergemeinschaft für angemessen gehalten werden, um die areale Variation mental in Einheiten zu gliedern und für gegebene Sprechanlässe zufrieden stellend kommunizierbar zu machen.

Folgende Datensammlungen sollen Anhaltspunkte zu dieser Fragestellung geben:

Aus einer Untersuchung aus den 1990er Jahren (vgl. Christen 1998) liegen Dialektbezeichnungen von Studierenden vor, die sie für ihren eigenen Dialekt (Tab. 1) als auch für jenen ihrer Mitstudierenden verwenden (Tab. 2). Ausserdem wurden die Mitarbeiter/innen des Schweizer Fernsehens DRS, die am Bildschirm als Moderator/innen auftreten, 2003 schriftlich dazu aufgefordert, anzugeben, welchen Dialekt sie sprechen (Tab. 3). Schliesslich wurde die COSMAS-Datenbank des Mannheimer IDS auf Dialektbezeichnungen hin ausgewertet. ${ }^{8}$ Das Schweizer Material, das sich aus den beiden Tageszeitungen Tages-Anzeiger und St. Galler Tagblatt konstituiert und damit eine deutlich ostschweizerische Prägung hat, wurde nach Kombinationen mit -deutsch-, -dialekt-, -mundart- (in verschiedenen Schreibweisen und mit unterschiedlichen Flexiven) abgefragt ${ }^{9}$ und liefert etwas mehr als 2000 Belege (Tab. 4). ${ }^{10}$

Die einzelnen Dialektbezeichnungen werden in den nachfolgenden Tabellierungen nach der Art ihrer Kategorisierungsniveaus unterschieden. ${ }^{11}$

7 Die alltagsweltlichen Kategorisierungen der Sprache betreffen nicht nur deren areale Dimension, sondern können auch die soziale Schichtung betreffen. Einer der befragten Fernsehmoderatoren (vgl. Tab. 3) bezeichnet seinen Dialekt als Baslerdeutsch und präzisiert ihn wie folgt: „'Strassendialekt', nicht ausgeprägt ,Daig' [Bezeichnung für Basler Oberschicht] und nicht ausgeprägt ,Rhyhafe', irgendwo zwischen den Dialektschlägen der beiden sozialen Pole Basels.“

8 Zur Zugänglichkeit verschiedener Korpora vgl. www.ids-mannheim.de/cosmas2/projekt/referenz/.

9 Es wird hier davon ausgegangen, dass Dialektkategorisierungen und -benennungen einerseits abhängig sind von der linguistischen Distanz zu sprachlichen Ausprägungen, andererseits aber auch vom soziokulturellen Stellenwert (arealer) sprachlicher Variation. Aus diesem Grunde ist es angebracht, die hier interessierenden Dialektbezeichnungen ausschliesslich in ihrem Vorkommen in der Deutschschweiz zu untersuchen.

10 Herzlichen Dank an meine Mitarbeiterin Mirjeta Reçi, die sich um die Beschaffung und Auswertung der COSMAS-Daten verdient gemacht hat.

11 Dass es sich bei Bezeichnungen wie Zürichdeutsch um Kategorisierungen auf dem Niveau von Kantonen und nicht von (gleichnamigen) Städten handelt, wird daran ersichtlich, dass es für letzteres die Bezeichnung Stadtzürcher Dialekt gibt. Der Dialekt der Städte Zürich, Basel, Bern, Luzern usw. kann dabei ohne Zweifel metonymisch für eine umfassendere natürliche Kategorie Zürichdeutsch usw. stehen, muss dies aber nicht. Vgl. auch Weber, bei dem der Dialekt der Stadt Zürich einen „Typus“ (1987: 24) des Zürichdeutschen darstellt, er aber als Referenzpunkt für das Zürichdeutsche seinen Idiolekt mit der Spielart „um Rüti-Hinwil“ auswählt. 
Tab. 1: Studierende: Bezeichnung des eigenen Dialekts (Christen 1998)

\begin{tabular}{|l|l|l|}
\hline Kategorienniveau & $\begin{array}{l}\text { Anzahl Nennungen } \\
\mathrm{N}=40\end{array}$ & Anzahl \% \\
\hline „Radio DRS“ & 1 & 2,5 \\
\hline $\begin{array}{l}\text { Typ: Landestei1 12 + Kanton } \\
\text { Beispiel: Ostschweizerisch St. Gallerdeutsch }\end{array}$ & 1 & 2,5 \\
\hline $\begin{array}{l}\text { Typ: Kanton } \\
\text { Beispiel: Zürichdeutsch }\end{array}$ & 23 & 57,5 \\
\hline $\begin{array}{l}\text { Typ: Kanton + Region } \\
\text { Beispiel: Berndeutsch Oberaargauer Dialekt }\end{array}$ & 4 & 10 \\
\hline $\begin{array}{l}\text { Typ: Region } \\
\text { Beispiel: Senslerdeutsch }\end{array}$ & 7 & 17,5 \\
\hline $\begin{array}{l}\text { Typ: Kanton + Ortschaft } \\
\text { Beispiel: Schwyzer Dialekt Einsiedlerdeutsch }\end{array}$ & 2 & 5 \\
\hline $\begin{array}{l}\text { Typ: Ortschaft } \\
\text { Beispiel: Winterthurer Dialekt }\end{array}$ & 2 & 5 \\
\hline
\end{tabular}

Tab. 2: Studierende: Bezeichnung der Dialekte der Kommiliton/innen (Christen 1998)

\begin{tabular}{|l|l|l|}
\hline Kategorienniveau & $\begin{array}{l}\text { Anzahl Nennungen } \\
\mathrm{N}=448\end{array}$ & Anzahl \% \\
\hline undefinierbar/unbekannt ${ }^{13}$ & 3 & 0,03 \\
\hline Typ: Landesteil & 14 & 3,2 \\
\hline Typ: Kanton & 417 & 95 \\
\hline Typ: Region & 14 & 3 \\
\hline Typ: Ortschaft & 0 & 0 \\
\hline
\end{tabular}

Tab. 3: Mitarbeiter/innen Fernsehen DRS: Bezeichnung des eigenen Dialekts (Befragung 2003; unveröffentlichtes Material) (=Antwort auf die Frage: Welchen Dialekt sprechen Sie?)

\begin{tabular}{|l|l|l|}
\hline Kategorienniveau & $\begin{array}{l}\text { Anzahl Nennungen } \\
\mathrm{N}=53\end{array}$ & Anzahl \% \\
\hline Oltener Bahnhofbuffet-Dialekt & 1 & $2 \%$ \\
\hline Typ: Landesteil & 1 & $2 \%$ \\
\hline Typ: Kanton & 42 & $79 \%$ \\
\hline $\begin{array}{l}\text { Typ: Kanton+Kanton } \\
\begin{array}{l}\text { Beispiel: Luzernerdeutsch mit Zürcher Dialekt } \\
\text { vermischt }\end{array}\end{array}$ & 5 & $9 \%$ \\
\hline Ortschaft & 4 & $8 \%$ \\
\hline
\end{tabular}

Tab. 4: COSMAS (Recherche 2008)

\begin{tabular}{|l|l|l|}
\hline Kategorienniveau & $\begin{array}{l}\text { Anzahl Nennungen } \\
\mathrm{N}=2088\end{array}$ & Anzahl \% \\
\hline
\end{tabular}

Stalder (1819: 273ff.) dokumentiert den Kanton Zürich anhand von zwei Übersetzungen, die eine als „Mundart in der Nähe der Stadt und um Kilchberg am See“, die andere als „Mundart um Ottenbach jenseits des Albis“ geltend.

12 Landesteil meint hier ein Areal, das die Grösse eines Kantons überschreitet (z. B. Ostschweiz), Region ein Areal, das diese unterschreitet und sich auf Täler (z. B. Rheintal), aktuelle oder ehemalige Verwaltungseinheiten (z. B. Entlebuch) oder Landschaften (z. B. Oberland) beziehen kann.

13 In drei Fällen geben die Befragten an, den Dialekt einer dritten Person nicht einordnen zu können. 


\begin{tabular}{|l|l|l|}
\hline $\begin{array}{l}\text { Typ: Deutschschweiz } \\
\text { Beispiel: Schweizerdeutsch }\end{array}$ & 827 & $40 \%$ \\
\hline Typ: Landesteil & 92 & $4 \%$ \\
\hline Typ: Kanton & 942 & $45 \%$ \\
\hline Typ: Region & 71 & $3 \%$ \\
\hline Ortschaft & 156 & $7 \%$ \\
\hline
\end{tabular}

Als Ergebnis dieser unterschiedlichen Recherchen kann konstatiert werden:

1. Das Kategorienniveau, das am häufigsten für Schweizer Dialekte verwendet werden, ist jenes nach der politischen Verwaltungseinheit Kanton.

2. Die Kantonsbezeichnungen werden gleichermassen für den Bezug auf Dialekte Dritter als auch für die Bezeichnung des eigenen Dialekts verwendet.

3. Neben dem Kategorienniveau Kanton kommen auch Bezeichnungen vor, die Areale denotieren, die grösser oder kleiner als Kantone sind.

Als erstes zeigt dieses Ergebnis, dass die areale Binnengliederung der Schweiz nach der politischen Verwaltungseinheit Kanton von herausragender alltagsweltlicher Relevanz ist. Die Kantonsdimension wird als taugliche und sinnstiftende Strukturierung arealer Heterogenität betrachtet, d. h. Kantone sind in der Schweiz Grössen, die weit über den Erfahrungsbereich Politik, Staatsgebilde hinaus als bedeutsam erachtet werden.

Roland Ris (1992: 756) erwägt, dass die Einteilung der Schweiz in Kantone einer soziokulturellen Gliederung der Schweiz gleichkommt. Die föderative Struktur der Schweiz mit Kantonen als in vielen Bereichen auch heute noch politisch autonomen Körperschaften mit „Fixpunkten in der Vergangenheit“ und ,symbolischen Figuren, an die sich die Erinnerung heftet" (Assmann 1992: 52) kann als Teil der kollektiven Erinnerung der Schweiz gesehen werden, der durchaus die Form eines ,kulturellen Gedächtnisses“ zukommt, wie es von Assmann (1992: 48ff.) definiert wird. Diese Kantone konstituieren Wahrnehmungsräume, die nach Ris (1992) den Dialektgliederungen vorgeordnet sind und eine mentale Organisationsstruktur bilden, welche die vorkommenden sprachlichen Verschiedenheiten entweder im Hinblick auf die Struktur einebnen oder aber herausstreichen kann (vgl. ähnlich auch Auer 2004).

\subsection{Kantonsdialekte als Basic-level-Kategorien}

Der Sachverhalt, dass Dialektbezeichnungen nach Kantonen mit Abstand den grössten Zuspruch erhalten, kann mit der besonderen Organisationsstruktur des dialektalen Wissens zusammenhängen und ein Indiz dafür sein, dass die dialektalen Kantonskategorien Basic-levelKategorien sind. Basic-level-Kategorien haben im Vergleich zu über- und untergeordneten Kategorisierungsniveaus eine prioritäre Stellung, die sich in Bezug auf vier Aspekte zeigt:

Perception: Overall perceived shape; single mental image; fast identification.

Function: General motor program.

Communication: Shortest ${ }^{14}$, most commonly used and contextually neutral words, first learned by children and first to enter the lexicon.

14 Was die Kürze der Bezeichnungen betrifft, so werden die Kantonsmundarten in den untersuchten Daten häufig mit dem Grundwort Deutsch (Zürichdeutsch, Baseldeutsch) bezeichnet, während dies beim Typ Region oder Ortschaft weit seltener der Fall ist. Diese Bezeichnungen sind nicht nur kürzer, sondern sie verleihen der jeweiligen Ausprägung gleichzeitig den Rang einer Sprache. 
Knowledge Organization: Most attributes of category members are stored at this level.

(Lakoff 1986: 47)

Kantonsbezeichnungen erweisen sich nicht nur als „most commonly used“, sondern die Kantonsdialekte zeigen auch hinsichtlich der Perzeption Eigenschaften von Basic-levelKategorien: So scheinen sich beispielsweise die Fernsehmitarbeiter/innen von einer Kantonsbezeichnung, mit der sie ihren Dialekt gegenüber unbekannten Dritten versehen, zu versprechen, dass diese mit einer einheitlichen Vorstellung verbunden werden kann.

Ein anderer Datentyp spricht ebenso für die Gestalthaftigkeit der Kantonsdialekte, nämlich die Personensignalemente, die für Such- und Vermisstenmeldungen verfasst werden und häufig auch Angaben zur Sprache der beschriebenen Person enthalten. Nur wenn nämlich für die Dialektkategorien Berner Dialekt und Zürichdeutsch - wie sie in den Signalementen in Beispiel $<1>$ und in Beispiel $<2>$ vorkommen - gilt, dass es für sie eine Vorstellung gibt und dass ihre Mitglieder eine Gestalt haben, die als gleich wahrgenommen wird, sind diese SprachSignalemente sinnvoll. Dass in einem Sprachsignalement die Kategorie Berner Dialekt (Beispiel $<1>$ ) überhaupt gewählt werden kann, geht von der Alltagserfahrung aus, dass eine bestimmte Zielgruppe über ein mentales (Laut-)Bild von Berner Dialekt verfügt. Dieses akustische Bild ermöglicht genauso wie die visuellen Bilder zur Grösse, zur Statur und zur Frisur eine Vorstellung der gesuchten Person. Wäre Zürichdeutsch (Beispiel <2>) ein Oberbegriff, so könnte man keine Vorstellung vom Idiolekt der genannten Person evozieren und die Angabe zum Dialekt würde sich von vorneherein erübrigen.

Beispiel $<1>$

Kinder im Lift belästigt

STADT BERN

(...) Wegen der Signalemente geht die Polizei vom selben Täter aus. Der Berner Dialekt sprechende, etwa 25-jährige Unbekannte ist 1,75 Meter gross und von hagerer Statur. Er hat magere Gesichtszüge und eine volle Unterlippe. Er trägt Rastalocken mit kurzem Rossschwanz im Nacken. Anfang Jahr trug er eine beige Jacke. Hinweise nimmt die Stadtpolizei Bern, Telefon 03132121 21, entgegen. (pd)

http://www.rabe.ch/medien/pdf_medien/02427Stadt20050129_1.pdf

Beispiel $<2>$

Zollikon. - Der 31jährige Fridolin Jud hat am Sonntag um 22 Uhr seinen Wohnort, das Wohnheim ,Stiftung zur Förderung geistig Invalider' an der Dufourstrasse, allein verlassen. Jud ist geistig behindert und hat Mühe, sich zu orientieren. Er ist $166 \mathrm{~cm}$ gross, hat blonde Haare, trägt eine Brille, eine rotbraune Wildlederjacke und spricht Zürichdeutsch. Hinweise über den Vermissten nimmt die Kantonspolizei (Tel. 01/247 22 11) entgegen. (E98/DEZ.32511 Züricher Tagesanzeiger, 16.12.1998, S. 25, Ressort: Region; IN KÜRZE)

Kantonsmundarten als volkstümliche Kategorien verfügen über eine lange Tradition ${ }^{15}$ : So lassen sich auch ältere Polizeisignalemente beibringen, in denen - wie heute - das dialektale Wissen offensichtlich auf diesem Niveau organisiert wird. In einem Signalementbuch aus der ersten Hälfte des 19. Jahrhunderts ${ }^{16}$ wird ein Färber aus den thurgauischen Romanshorn sprachlich wie folgt charakterisiert: „er führt mehr den Berner- als den Thurgauer-Dialekt, spricht auch französisch“. Über eine Näherin und Kurzwarenhändlerin wird vermerkt, sie hätte „eine

15 Vgl. oben Franz Joseph Stalders Kantonsmundarten (1819).

16 Vgl. Allgemeines Signalement-Buch für die Schweizerische Eidgenossenschaft, enthaltend alle ab Seite der 19 Hohen Stände verhängten öffentlichen Verhafts-Befehle, Steckbriefe, Landesverweisungen, Beschreibungen und Warnungen vor verdächtigen Individuen, Anzeigen von beträchtlichen Diebstählen etc. 19 Bde. Bern 18091848; hier zitiert nach Furrer (2002). 
grobtönige, schnelle Aussprache, sonst höflich, spricht den Luzernerdialekt, daneben spricht sie auch geläufig französisch."

\subsection{Oberbegriff oder Basic-level-Kategorie?}

Auffällig ist, dass die Kategoriengrösse Ostschweiz (z. B. Ostschweizer Dialekt, Ostschweizer Mundart) in den COSMAS-Daten - auch im Vergleich zu Bezeichnungen ostschweizerischer Kantone - sehr häufig belegt ist:

Tab. 5: Quantitatives Vorkommen der Kategoriengrösse Ostschweiz

\begin{tabular}{|l|l|}
\hline Appenzell & 90 \\
\hline Ostschweiz & 87 \\
\hline St. Gallen & 62 \\
\hline Graubünden & 32 \\
\hline Innerrhoden & 28 \\
\hline Thurgau & 20 \\
\hline
\end{tabular}

Die Dialektbezeichnung wird in einigen Fällen auch für den eigenen Dialekt verwendet (eine Fernsehmoderatorin bezeichnet ihren Dialekt als Ostschweizerdialekt). Wie die Kantonsbezeichnungen, so wird auch die Kategoriengrösse Ostschweiz für Signalemente verwendet:

Beispiel $<3>$

Bei jedem der Diebstähle ergaunerte sich der Mann mehrere hundert Franken Bargeld. Die Polizei sucht einen 20 bis 25-jährigen Mann, zirka 170 Zentimeter gross und schlank, er hat kurze dunkle Haare, wird als südländischer Typ beschrieben, der Ostschweizer Dialekt spricht. uwf. (A01/JUL.15919 St. Galler Tagblatt, 06.07.2001; Der Schreck der Serviertöchter)

Dem Kategorienniveau Ostschweiz scheint der gleiche Rang zuzukommen, wie er oben für die Kantonsbezeichnungen erläutert wurde, und nicht etwa eine übergeordnete Klassifizierungsebene zu etablieren. Kategorienbezeichnungen vom Typ Landesteil, die Züge einer Basic-levelKategorie tragen, sind in COSMAS nur für die Grösse Ostschweiz ausgewiesen. Um der Frage nachzugehen, ob die Kategoriengrösse Ostschweiz nicht einfach der speziellen Quellenlage von COSMAS geschuldet ist, wurde eine Gegenprobe im Schweizer Text Korpus (http://www.schweizer-textkorpus.ch/) vorgenommen, das für Landesteil + Dialekt/Mundart/Deutsch 9 Belege ausweist, die ausnahmslos die Ostschweiz betreffen. Die Suchmaschine Google (25.9.2008, Schweizer Seiten) bestätigt das überaus häufige Vorkommen und damit den Sonderstatus von Dialektbezeichnungen mit dem Determinans Ostschweiz:

Tab. 6: Quantitatives Vorkommen von Kategorienbezeichnungen Typ Landesteil

\begin{tabular}{|l|l|}
\hline Stand: 25.9.08, CH-Seiten & \\
\hline Ostschweiz & 357 \\
\hline Westschweiz & 6 \\
\hline Nordschweiz & 0 \\
\hline Innerschweiz & 40 \\
\hline Mittelland & 11 \\
\hline Zentralschweiz & 4 \\
\hline
\end{tabular}


Die vergleichsweise selten - und weder als Signalement noch als Eigenbezeichnung - belegte Kategorienbezeichnung Innerschweizer Dialekt scheint ebenfalls tauglich zu sein, um in der Deutschschweizer Sprechergemeinschaft - aber wohl ausserhalb der Innerschweiz selbst - eine gestalthafte Vorstellung zu evozieren:

Beispiel $<4>$ :

Markus Burch, dipl. Bergführer

Pflichtbewusstsein, Ruhe und Gelassenheit zeichnen Markus aus. Ein filigraner Kletterer, der mit seinem Innerschweizer-Dialekt unsere Gäste in seinen Bann zieht und das ,Buttermaitli' aus der Werbung in den Schatten stellt. http://www.bergundtal.ch/index_team.html (23.4.2008)

Als Erklärungen für die Existenz der Kategorie Ostschweiz kommen sowohl soziokulturelle Aspekte als auch Kontrastarmut zwischen den arealen Varietäten in Frage. Entweder haben die ostschweizerischen Kantone als Einzelkantone eine $\mathrm{zu}$ geringe Bedeutung, als dass es sozialpsychologisch wichtig wäre, sie auch (sprachlich) auseinander halten zu können oder aber ihre arealen Varietäten sind sich - immer aus der Perspektive der Deutschschweizerinnen und Deutschschweizer - derart ähnlich, dass sie als gleich wahrgenommen werden. Das könnte durchaus auch für die Innerschweizer Dialekte zutreffen, was durch die Existenz des Verbs länderen (Id. III, 1310) untermauert wird, das die Bedeutung ,in Sprache und Sitten den Bewohner der ,Länder' verraten“ hat, wobei mit Länder die Urkantone oder gar die Fünf Orte ${ }^{17}$ gemeint sind.

Einen entschieden anderen Status als Kantonsdialekte resp. die Kategorie Ostschweizerisch hat die Kategorie vom Typ Deutschschweiz, die offensichtlich nicht dazu taugt, die idiolektale Ausprägung eines Deutschschweizer Dialektes zu erfassen. Bezeichnenderweise kommt dieser Kategorientyp denn hier auch ausschliesslich in den COSMAS-Belegen vor. Für den Gebrauch dieses Kategorientyps ist charakteristisch, dass er vornehmlich dann zum Zuge kommt, wenn generalisierend auf beliebige Dialekte Bezug genommen wird:

Beispiel $<5>$

Doch inzwischen sind auch bei andern grossen Parteien Bestrebungen im Gang, in der Ausländerpolitik das Feld nicht nur der SVP zu überlassen. So haben die meisten ebenfalls Grundsatzpapiere verabschiedet und Vorstösse lanciert. Jüngstes Beispiel ist die FDP. Sie reicht diesen Mittwoch im Stadtparlament neun Vorstösse ein, die nach einer härteren Gangart rufen. Verlangt wird unter anderem, dass der Stadtrat beim Bund auf eine neue Gesetzgebung der Niederlassung von Ausländern und auf eine Senkung der Fürsorgeleistungen drängt. Zudem möchte die FDP den Ausländeranteil in städtischen Wohnungen auf 25 Prozent begrenzen und Asylbewerber auch gegen ihren Willen etwa zur Stadtreinigung einsetzen. Eine Verschärfung fordern die Freisinnigen auch bei der Einbürgerung. Die Wohnsitzfrist von sechs Jahren wollen sie beibehalten, dafür aber Schweizerdeutsch, staatsbürgerliche Kenntnisse und wirtschaftliche Selbsterhaltung stärker gewichten. (E99/JUN.14598 Züricher Tagesanzeiger, 01.06.1999, S. 15, Ressort: Zürich und Region; Neue SVP-Initiative gegen Einwanderung)

Auch für nicht-authentische, lernersprachliche Dialekte werden Kategorisierungen vom Typ Deutschschweiz verwendet:

Beispiel $<6>$

Einer der unbekannten Männer ist etwa 30 Jahre alt, rund 1,75 Meter gross, hat eine längliche Kopfform und ist von schlanker Statur. Zur Tatzeit trug er laut Kantonspolizei eine gestrickte, dunkelbraune Strumpfmaske mit zwei

17 Die Fünf Orte Uri, Schwyz, Unterwalden, Zug und Luzern bildeten bis zum Ende des Ancien régime den Kern der katholischen Eidgenossenschaft. 
Sehschlitzen, ein beiges Hemd mit dunklen Längsstreifen und dunkelblaue Hosen. Der Mann war mit einem Gewehr bewaffnet und sprach gebrochen Schweizerdeutsch. (A97/SEP.22936 St. Galler Tagblatt, 09.09.1997; Hafenbahnhof Kreuzlingen erneut überfallen)

Es sei betont, dass die Art, wie die sprachliche Wirklichkeit in der Deutschschweiz alltagsweltlich erfasst und kategorisiert wird, durch die spezifischen sozialen Bedingungen der Lebenswelt geprägt ist. Eine Grösse wie Schweizerdeutsch, die für die Deutschschweizerinnen und Deutschschweizer einer generalisierenden Ebene ohne einheitliche Vorstellung angehört, mag aus anderer Perspektive eine Kategorie der Basisebene sein, wie sich das in folgendem Beleg für Schweizer Dialekt andeutet, der von einer bundesdeutschen Webseite stammt.

Beispiel $<7>$

Für unsere Schweizer-User haben wir hier etwas ganz Besonderes: Sprüche für alle Lebenslagen im typischen Schweizer Dialekt - wenn das nicht zur ...

www.livinghandy.de/sms-sprueche-kat.html

\subsection{Basic-level-Kategorie oder Ebene der Spezialisierung?}

Was die Kategorisierungstypen Region und Ortschaft betrifft, so sind diese insgesamt seltener als die Kantonsbezeichnungen; sie werden kaum zur Bezeichnung von Dialekten Dritter verwendet (vgl. Tab. 2), häufiger dagegen zur Bezeichnung des eigenen Dialekts. Charakteristisch für den Gebrauch dieses Kategorientyps in COSMAS - nachfolgend illustriert anhand von Dialektbezeichnungen mit Namen von Tälern (Beispiel $<8>$ ), Landschaften (Beispiel $<9>$ ) und Ortschaften (Beispiel $<10>$ ) - ist, dass hier insofern von einem markierten Kontext ausgegangen werden kann, als der Text auf Lokales und/oder betont Lustiges/Alltägliches fokussiert und dadurch Dialektstereotypen aktiviert. $\mathrm{Zu}$ diesem tendenziell folkloristischen und vergangenheitsorientierten Zugriff auf Lokales gehört, dass im Dialekt zitiert (Beispiel $<8>$ ) wird, ${ }^{18}$ oder dass die Dialektbezeichnung selbst in Dialekt wiedergegeben wird (Beispiel $<10>$ ).

Beispiel $<8>$

Ein älterer Herr mit Glatze und grauem Haarkranz hat den Vorfall ebenfalls bemerkt. Seine Gesichtsfarbe rötet sich unmerklich, dann platzt ihm der Kragen und in unüberhörbarem Rheintaler Dialekt weist er die Frau zurecht: ,Wi kama o so ibildat tua, da spielt doch wörklich ku Rolla, öb eras Füüdla hockad oder seazad. Bi üs setma o hocka, das isch schliesslich üsara Dialäkt, und wämmer e soa redid, seammer wäge deam no lang ku Rüppil. I wür mi schämma, deam Buab e soa ko -- und äs wür i mi’ bekräftigt er seine Zurechtweisung. Von den übrigen Mitfahrenden hätte sich ja wohl kaum jemand so eine Rüge erlaubt, die Blicke aber verrieten um so eifrigere Zustimmung. (A98/AUG.51947 St. Galler Tagblatt, 12.08.1998; Seitenblick)

$<$ Beispiel 9>

Und so entstand 1989, sozusagen als Nebenprodukt der Chronik, sein erstes Buch ,S Gwönderbüechli', wo der Autor in prägnantem Kurzenberger Dialekt lustige Begebenheiten und humorvolles Geschehen aus dem Alltag seiner Mitmenschen Revue passieren lässt. Das Echo war gross, und dem Schriftsteller wurden immer wieder neue Geschichten zugetragen. So sind bis jetzt vier Mundartbüchlein entstanden. Das fünfte erscheint in diesen Tagen (siehe Kasten). 'Es ist mir ein Anliegen, der Nachwelt Geschichten zu erhalten, die sonst mit ins Grab genommen worden wären', erklärt Peter Eggenberger, ,und nur in Mundart sind sie wirklich echt.' (A99/OKT.72249 St. Galler Tagblatt, 15.10.1999; Heiteres in Kurzenberger Dialekt)

$<$ Beispiel 10>

18 Das Beispiel $<8>$ illustriert gleichzeitig den in südlichen Regionen des deutschen Sprachgebietes verbreiteten Sprachwitz, der die Überlegenheit der Dialekte zum Thema hat (vgl. Niebaum \& Macha 2006: 198ff.), wobei es hier nicht um den Gegensatz zwischen Dialekt und Standardsprache geht, sondern um den kleinräumigen Dialekt eines Tales, in dem sich - so die Vorstellung - die angemessenen Worte finden lassen. 
Degersheim. 'Weisch no?' ist das Thema des Seniorennachmittags vom Mittwoch, 11. November, 14.30 Uhr im katholischen Pfarreiheim Degersheim. Dölf Nef wird in unverfälschtem 'Tegerscher' Dialekt mit Episoden aus dem Dorfgeschehen aufwarten. Musikalisch wird der Anlass von einer Flötengruppe aus Magdenau begleitet. pd. (A98/OKT.68915 St. Galler Tagblatt, 30.10.1998; 'Weisch no?' mit Dölf Nef)

Der Gebrauch dieses Kategorientyps in den COSMAS-Belegen macht deutlich, dass diese zu einer Ebene der Spezifikation gehören. Die Bezeichnungen selbst lassen sich dahingehend interpretieren, dass Dörfer und Talschaften alltagsweltlich als soziale Orte verstanden werden, deren Zugehörige sich durch sprachliche Gemeinsamkeiten auszeichnen. Die Unterschiede zur unmittelbaren dialektalen Nachbarschaft werden also durchaus wahrgenommen und können nachweislich zu Neckereien und Spott führen, allerdings wird erst im Fachgespräch auf diese Eigenheiten eingegangen. Dass bei den Fernsehmoderator/innen vier ihren zürcherischen Dialekt nach der Stadt Winterthur bezeichnen (vgl. Tab. 3), dürfte mit der innerzürcherischen Konkurrenz der beiden Städte Zürich und Winterthur zu tun haben: Die Betonung der Eigenständigkeit gegenüber der Grossstadt Zürich, kann ihren Ausdruck (auch) in der Hervorhebung weniger dialektaler Unterschiede finden und die Wahl des Spezifikationstyps im vorliegenden Befragungskontext angemessen erscheinen lassen.

Bei der alltagsweltlichen Kategorisierung von Dialekten, wie sie sich in der Wahl und im Gebrauch von Dialektbezeichnungen manifestiert, zeigen sich jene Eigenheiten, die bei natürlicher Kategorienbildung zu erwarten sind. Obwohl keine eigentlichen psychologischen Experimente vorliegen, stützen vor allem der Sachverhalt der in den am häufigsten verwendeten Dialektbezeichnungen manifest werdenden Kategorisierungsniveaus und der Sachverhalt der Verwendung von Dialektbezeichnungen bestimmter Kategorisierungsniveaus für Signalemente die Hypothese, dass es in der Deutschschweiz unterschiedliche dialektale Kategorisierungsebenen gibt, die nicht gleichrangig sind, sondern dass das alltagsweltliche Dialektwissen in der Regel auf der Ebene Kanton mental organisiert wird:

Abbildung 1: Hierarchie von Kategorisierungsebenen

\begin{tabular}{|l|l|l|l|}
\hline $\begin{array}{l}\text { Übergeordnete Ebene: } \\
\text { Generalisierung }\end{array}$ & Tier & Pflanze & Schweizerdeutsch \\
\hline $\begin{array}{l}\text { Basisebene: } \\
\text { Basic-level-Kategorien }\end{array}$ & Hund / Katze & Blume / Baum & $\begin{array}{l}\text { Zürichdeutsch / } \\
\text { Ostschweizer Dialekt }\end{array}$ \\
\hline $\begin{array}{l}\text { Untergeordnete Ebene: } \\
\text { Spezifizierung }\end{array}$ & Pudel / Siamesenkatze & Gladiole / Buche & $\begin{array}{l}\text { Rheintaler Dialekt / } \\
\text { Kurzenberger Dialekt / } \\
\text { Tegerscher Dialekt }\end{array}$ \\
\hline
\end{tabular}

Das Ergebnis untermauert insgesamt nicht nur, dass es in der Deutschschweiz ein virulentes Bedürfnis nach Kategorisierung der arealen Varianz gibt, sondern auch, dass die areale Varianz mental nicht etwa ausgeblendet und beispielsweise als gestalthaftes Schweizerdeutsch konzeptualisiert wird, sondern die areale Varianz wird aus der Perspektive der Deutschschweizerinnen und Deutschschweizer als binnenschweizerisch untergliedert erfahren.

\section{Alltagsweltliche Dialektattribuierungen}

Sollte es sich - wie angenommen - bei den alltagsweltlichen Dialektkategorien um natürliche Kategorien handeln, so darf mit so genannten Prototypeneffekten gerechnet werden resp. bestimmte Phänomene, die sich im Zusammenhang mit Dialektkategorien zeigen, können das Urteil stützen, dass es auch in Bezug auf das Objekt Sprache natürliche Kategorienbildung gibt. 
Die Belege aus dem COSMAS-Korpus werden nun darauf hin untersucht, inwiefern Modifikationen, die Dialektbezeichnungen attribuieren, nicht nur der allgemeinen sozialen Wertorientierung dienen und etwa zeigen, welche Dialekte in der Sprechergemeinschaft als schön oder hässlich gelten, sondern die Zugehörigkeit zu einer Kategorie qualifizieren und damit deren „centrality gradience“ bestätigen, nämlich ,the idea that members (or subcategories) which are clearly within the category boundaries may still be more or less central" (Lakoff 1986: 12).

Die Wortwahl, die für die Qualifizierung von Dialekten herangezogen wird, soll überdies auf metaphorische Übertragungen hin besehen werden und dadurch einen Schlüssel liefern zur alltagsweltlichen Konzeptualisierung von Dialekt(en).

\subsection{Prototypeneffekte in Form von Dialektattribuierungen}

Von den 2088 Kategorienbezeichnungen, die in COSMAS aufgefunden wurden, werden 296 meist adjektivisch attribuiert, d. h. ungefähr jede siebte Kategorienbezeichnung erfährt eine zusätzliche semantische Modifikation. ${ }^{19}$ Bei den Kategorisierungen vom Typ Kanton und Region ist es dabei etwa jede vierte Bezeichnung, bei den Bezeichnungen des Typs Landesteil und Ortschaft nicht ganz jede fünfte Bezeichnung, die attribuiert wird. Anders dagegen die Bezeichnungen des Typs Deutschschweiz, von denen nur etwa jede hundertste attribuiert wird. Diese Zahlenverhältnisse sind ein deutliches Indiz dafür, dass es sich beim Kategorisierungstyp Deutschschweiz um die Ebene der Oberbegriffe handelt. Das Bedürfnis, solche Bezeichnungen in irgendeiner Weise zu modifizieren, ist zwangsläufig seltener, als bei Bezeichnungen, die mit einem mentalen Hörbild verbunden sind.

Tab. 7: Dialektattribuierungen und Kategorisierungsniveaus

\begin{tabular}{|l|l|l|l|l|l|l|}
\hline $\begin{array}{l}\text { Kategorisierungs- } \\
\text { niveau }\end{array}$ & $\begin{array}{l}\text { Typ } \\
\text { Deutsch- } \\
\text { schweiz }\end{array}$ & $\begin{array}{l}\text { Typ } \\
\text { Landesteil }\end{array}$ & $\begin{array}{l}\text { Typ } \\
\text { Kanton }\end{array}$ & $\begin{array}{l}\text { Typ } \\
\text { Region }\end{array}$ & $\begin{array}{l}\text { Typ } \\
\text { Ortschaft }\end{array}$ & Total \\
\hline $\begin{array}{l}\text { TOTAL } \\
\text { Attribuierungen }\end{array}$ & 9 & 17 & 227 & 20 & 23 & 296 \\
$\begin{array}{l}\text { TOTAL Dialekt- } \\
\text { Bezeichnungen } \\
\begin{array}{l}\text { Relative Häufigkeit } \\
\text { von Attribuierungen }\end{array}\end{array}$ & 827 & 92 & 942 & 71 & 156 & 2088 \\
\hline
\end{tabular}

Zur Attribuierung der Dialektbezeichnungen werden 86 unterschiedliche Lexeme verwendet, 54 davon sind bloss ein Mal belegt. Nimmt man jene Lexeme in den Blick, die 50\% der vorkommenden Tokens umfassen, so sind dies die folgenden Adjektive ${ }^{20}$ :

Tab. 8: Häufigste Dialektattribute

19 Zur Leistung von Adjektiven schreibt Weinrich (2005: 509): „Sie können eine Quantität oder eine Qualität, eine Relation oder eine Klassenzugehörigkeit bezeichnen, sie können zur Orientierung oder Dimensionierung, zur Charakterisierung oder Situierung, zur Beschreibung oder Unterscheidung, zur Einschätzung oder Beurteilung und zu manchem anderen dienen."

20 Komparationsformen werden zum Adjektiv im Positiv gerechnet, Determinativkomposita zum Grundwort. 


\begin{tabular}{|l|l|}
\hline breit, breitest- & 56 \\
\hline urchig, urchigst- & 22 \\
\hline rein, lupenrein, astrein, reinst- & 19 \\
\hline schön, schönst & 16 \\
\hline echt, waschecht & 15 \\
\hline ausgeprägt & 15 \\
\hline unverkennbar & 12 \\
\hline unverfälscht & 10 \\
\hline typisch & 9 \\
\hline
\end{tabular}

Das am häufigsten belegte Adjektiv breit, breitest (56 v. 296) wird einerseits zur absoluten Qualifizierung eines bestimmten Dialekts verwendet:

Beispiel $<11>$

Die Geschichten von Ueli Bietenhader bedeuten Heimat. Handlungsorte und Personen stimmen, erinnern die ebenfalls in Altstätten aufgewachsenen Leser oder Zuhörer an die eigene Vergangenheit und lassen so eigene Geschichten wieder lebendig werden. Dazu trägt auch der breite Altstätter Dialekt bei, der irgendwo zwischen dem Diepoldsauer 'moana' und dem Appenzellischen 'meene' liegt, wie Ueli Bietenhader 'mänt' - oder meint. (A99/NOV.80236 St. Galler Tagblatt, 15.11.1999; Geschichten aus der Altstätter Jugend)

Andererseits wird mit breit auch eine bestimmte idiolektale Ausprägung eines Dialektes qualifiziert. Diese Gebrauchsweise impliziert dann die Existenz von Vergleichsgrössen, die nichtbreites Berndeutsch, nicht nicht-breites Zürichdeutsch usw. sind. Diese relativierende Lesart resultiert aus einer natürlichen Kategorienbildung, die bei elativ verwendetem breitest- besonders deutlich wird:

Beispiel $<12>$

Die stets gut besuchte, Bodega' bietet noch immer die hier zu Lande eher seltene Gelegenheit, sozusagen niederschwellig mit einem auf den ersten Blick nicht unsympathischen Subjekt ins Gespräch zu kommen, das selbstverständlich nicht zwingend gleich erotisches Objekt zu sein braucht. Es genügt eine Zürcher Tageszeitung neben Jérez und Tapas liegen zu haben, um schon nach wenigen Minuten in intensivste journalistische Qualitätsdebatten verwickelt zu sein. ,Setzt man sich an den richtigen Tisch, ist der Abend schon gerettet', sagt der Internet-Designer vis-à-vis, der die Ansicht vertritt, heute gehe es keiner Zeitung mehr um Qualität, sondern , lediglich ums Geldmachen', während die Dame nebenan in breitestem Bärntütsch zwar druckerschwarz, aber nicht schwarz sieht: ,Zeitungen werden nie verschwinden! Ich könnte nie ohne sein!' Nie verschwinden wird in der ,Bodega' jedenfalls der Rauch, ohne den wir zwar durchaus sein könnten, der aber wie die seit Jahrzehnten bedienenden Kellner unbedingt dazugehört. (E00/MAR.07836 Züricher Tagesanzeiger, 21.03.2000, S. 18, Ressort: Stadt Zürich; Geselligkeit bei Jérez und Tapas)

Ein Indiz dafür, dass diese relativierende Lesart auch bei ambigem Gebrauch von breit häufig intendiert sein könnte, ist dessen uneingeschränkter Gebrauch für verschiedene Dialektausprägungen: Da breit nämlich in den Daten von COSMAS mit Dialektbezeichnungen vorkommt, die sich auf Appenzell, Basel, Bern, Graubünden, Innerrhoden, Luzern, St. Gallen, Uri, Zürich, die Schweiz, die Ostschweiz, das Emmental und die Ortschaften Altstätten und Diepoldsau beziehen, scheint breit weniger einen bestimmten Dialekt zu charakterisieren als einzelne Idiolekte (im Unterschied dazu ist beispielsweise hell nur für St. Gallen, gemütlich nur für Bern belegt).

Die Erwägung, ob es sich um die absolute Wertung eines Dialekts oder um die relativierende Wertung eines Idiolekts in Bezug auf eine Vergleichsgrösse handelt, gilt auch für urchig, schön und unverkennbar. Ausschliesslich die relativierende Lesart - und damit ein Prototypeneffekt ist im Spiel bei den Adjektiven rein (Beispiel $<13>$ ), echt (Beispiel $<14>$ ), ausgeprägt (Beispiel 
$<15>$ ), unverfälscht (Beispiel <16>) und typisch (Beispiel <17>).

Beispiel $<13>$

Der 45minütige Spaziergang durch das prächtige Dorf, Träger des Wakker-Preises, was seine Bewohner und Bewohnerinnen gerne betonten, war beschaulich, wie es sich für einen vorabendlichen Bummel auch gehört. Star war eindeutig Martha Schmid, gebürtig aus Fiesch, Gattin des Präfekten. Sie begleitete Moderator Tschuor kundig und mit einer guten Portion Mutterwitz durch die Gemeinde, half bei Verständigungsschwierigkeiten zwischen seinem reinen Bündner Dialekt und dem ebenso reinen Walliser ,Diitsch' der Ernerinnen und Erner. (E97/JUL.17643 Züricher Tagesanzeiger, 22.07.1997, S. 12, Ressort: Kehrseite; Gang durchs Dorf)

Beispiel $<14>$

$</$ BYLINE $>$ Die Überraschung war gross, als die Bündner SVP für die Nationalratswahlen die Kandidatur der ehemaligen SVP-Generalsekretärin Myrtha Welti-Hunger bekannt gab. Die 54-jährige Botschaftergattin spricht zwar echten Bündner Dialekt, hat ihren Wohnsitz aber in der Stadt Zürich. (A99/AUG.57181 St. Galler Tagblatt, 21.08.1999; Myrtha Welti- Hunger)

Beispiel $<15>$

Dass er das Baselbiet noch heute liebt, braucht er nicht besonders zu betonen. Allein sein ausgeprägtes Baseldeutsch verrät, dass er nie aufhören wird, ein Basler zu sein. Die Zugehörigkeit zu einer Basler Fasnachtsclique, seine Tätigkeit als Larvenbildner und die Tatsache, dass er in den dreissig Jahren seiner Thurgauer Zeit die Teilnahme an der Basler Fasnacht nie verpasst hat, sprechen eine deutliche Sprache. Nichtsdestotrotz gefällt es Ernst Friedli hier ausgezeichnet. Er habe die Thurgauerinnen und Thurgauer als fröhliche, offene Menschen kennen und schätzen gelernt. (A99/JAN.02902 St. Galler Tagblatt, 15.01.1999; Heimweh-Basler fühlt sich wohl hier)

Beispiel $<16>$

Die am Telefon mit ihrem unverfälschten Bündner Dialekt noch sehr regsam wirkende Mutter Reto Brauns erinnert sich, dass ihr Sohn recht intensive Kontakte mit Tischhausers Buben pflegte, was Vater Anton Tischhauser bestätigt. 'Ja, er war viel bei uns zuhause. Ihn zeichnete schon damals hohe Intelligenz aus, aber er blieb immer bescheiden. Dass er es so weit gebracht hat, verdankt er seinem gesunden Menschenverstand und einem intensiven Selbststudium. Ich jedenfalls habe ihn in bester Erinnerung.'(A98/JUN.41630 St. Galler Tagblatt, 22.06.1998; Als 'sauglatte Bueb' im Gedächtnis)

Beispiel $<17>$

Am Dienstag, 22. August, $14 \mathrm{Uhr}$, gibt Peter Eggenberger im katholischen Pfarreiheim Herisau einen kleinen Einblick in den typischen Appenzeller Dialekt und liest aus verschiedenen Mundartbüchern vor. Die Besucher werden in gemütlicher Runde einen humorvollen Nachmittag erleben, denn Peter Eggenberger wird über bekannte Persönlichkeiten und Originale, die im Vorderland gewirkt und gelebt haben, berichten und erzählen. Gleichzeitig können Programme für den Halbtagesausflug vom 19. September auf die Alp Malbun mit einem Zvierihalt gekauft werden. pd. (A00/AUG.54539 St. Galler Tagblatt, 18.08.2000; Peter Eggenberger beim Club der Älteren)

Mit den in den Beispielen $<13>$ bis $<17>$ illustrierten Attribuierungen wird jeweils die Zugehörigkeit zu einer Kategorie bewertet. Die genannten Adjektive qualifizieren einzelne Dialektausprägungen als herausragend und implizieren gleichzeitig die Existenz von Ausprägungen, denen dieses (positive) Urteil nicht zukommt.

Die Adjektive, die eine einzelne Ausprägung im Hinblick auf andere Ausprägungen desselben Dialekts qualifizieren, sind als Effekte zu beurteilen, die aus der Prototypenstruktur der alltagsweltlichen Dialektkategorisierung resultieren, bei der zwischen besseren und schlechteren, eindeutigeren und weniger eindeutigen Zugehörigkeiten unterschieden wird.

Dass sich in COSMAS keine Belege für Hedges wie eine Art von Zürichdeutsch oder irgendwie Berndeutsch finden, die ebenfalls auf natürliche Kategorienbildung mit unterschiedlicher Zentralität hinweisen würden, ist dem Sachverhalt geschuldet, dass idiolektale Dialektausprägungen in den Schweizer Printmedien häufiger charakterisiert werden, wenn sie den Anforderungen an ein Dialektideal besonders gut entsprechen - eher prototypische als periphere Kategorienmitglieder sind erwähnenswert. Bei den Eigenbezeichnungen, wie sie in den 
Korpora belegt sind, kommt bei peripherer schlechter Kategorienzugehörigkeit das Bedürfnis nach einer Präzisierung ins Spiel, von der Art Luzernerdeutsch mit Zürcherdialekt vermischt. Hedges von der Art so etwas wie Luzerndeutsch könnten jedoch bei der Einschätzung von Hörproben erwartet werden.

\subsection{Attribuierungen als Indikatoren für alltagsweltliche Dialektkonzepte}

Die Wahl der Adjektive, die zum Zuge kommen, um Sprachliches zu qualifizieren, zeigen gleichzeitig auf, wie der Erfahrungsbereich Dialekt konzeptualisiert wird. Nur am Rande kommen Adjektive ins Spiel, die direkt mit der Produktion und Perzeption von Sprachen als Lauterzeugungen zu tun haben (wohlklingend, nichtsingbar, unüberhörbar, nasal). In der Regel wird anhand der Attribuierungen deutlich, dass Konzepte anderer Erfahrungsbereiche metaphorisch oder metonymisch übertragen und auf den Bereich des Dialekts projiziert werden. Die Übertragungen stellen einen Weg dar, um die ephemeren, in der Zeit ablaufenden Sprechereignisse, die ja der menschlichen Wahrnehmung als Einziges überhaupt zugänglich sind, fassbar zu machen.

Für einige der attribuierenden Adjektive lassen sich konzeptionell grundlegendere, konkretere Bereiche angeben, die als Ausgangsbereiche oder Bildspender (source domains) zur Erfassung sprachlicher Grössen (target domain) dienen (vgl. zur Theorie der Metapher im Rahmen der kognitiven Semantik Saeed 1997: 345ff.):

Abbildung 2: Dialektattribute als semantische Übertragungen

\begin{tabular}{|c|c|c|}
\hline & $\begin{array}{l}\text { Source domain } \\
\text { Ausgangsbereich }\end{array}$ & $\begin{array}{l}\text { Target domain } \\
\text { Zielbereich }\end{array}$ \\
\hline & $\begin{array}{l}\text { Geometrischer Körper } \\
\text { breit, spitz, rund, eckig... }\end{array}$ & \multirow{5}{*}{ Dialekt } \\
\hline & $\begin{array}{l}\text { Artefakt } \\
\text { geschliffen, urchig?, gemütlich?... }\end{array}$ & \\
\hline $\begin{array}{l}\frac{\overrightarrow{0}}{0} \\
\frac{0}{0} \\
\Xi\end{array}$ & $\begin{array}{l}\text { Stoff } \\
\text { echt, waschecht, rein, unverfälscht, farbig, sauber, } \\
\text { verwaschen, X-eingefärbt, gemischt, mit X- } \\
\text { Einschlag,... }\end{array}$ & \\
\hline \multirow[b]{2}{*}{ 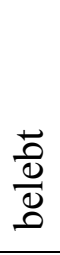 } & $\begin{array}{l}\text { Organismus } \\
\text { alt, lebendig, flink... }\end{array}$ & \\
\hline & $\begin{array}{l}\text { Mensch } \\
\text { sympathisch, witzig, derb, vornehm, anbiedernd.... }\end{array}$ & \\
\hline
\end{tabular}

Bei der Mensch-Dialekt-Übertragung ist statt an metaphorische Übertragung auch an Metonymie zu denken: Die Charakterisierung eines Äusserungsgehalts als witzig oder anbiedernd wird auf die Äusserungsform übertragen.

Die Zuweisungen, die hier gemacht werden, sind alles andere als unproblematisch. Einerseits lässt sich bei einigen Adjektiven wie etwa urchig oder gemütlich nicht unmittelbar entscheiden, zu welchem Ausgangsbereich sie gehören. Das ist dem Umstand geschuldet, dass zwischen 
diesen Ausgangsbereichen mit semantischen Übertragungen gerechnet werden muss, deren Ausgangs- und Zielbereich synchron nicht mehr ersichtlich sind. Ausserdem ist immer zu fragen, welches Stadium in einer mehrstufigen semantischen Übertragungskette überhaupt als Bildspender anzusetzen ist. Sprachliche Charakterisierungen wie holpriges Berndeutsch oder holprige Sprache mögen konventionalisiert und tote Metaphern sein (zur Wortgeschichte von holprig vgl. Paul 2002). Entscheidend ist, dass gerade die häufig belegten attribuierenden Adjektive einen Einblick vermitteln in den Dialektdiskurs, der mit (positiven) Metaphern aus dem Stoffbereich wie rein, sauber, unverfälscht von einer Dialektideologie zeugt, wie sie sonst nicht für Dialekte sondern für Standardsprachen üblich ist. ${ }^{21}$ Was die seltener belegten Adjektive betrifft, so wird ersichtlich, dass für den Zielbereich Dialekt nach wie vor die gleichen Bildspender wirksam zu sein scheinen: Gerade im Korpus selten oder sogar singulär belegte Dialektattribuierungen wie verwaschen oder saftwurzlig zeigen, dass für die Charakterisierungen dialektaler Ausprägungen mehrheitlich Modifikationen verwendet werden, welche die Dialekte mit zwei Bildbereichen in Verbindung bringen: Dialekt als unbelebte Materie und - im vorliegenden Korpus weit seltener - Dialekt als belebter Organismus. ${ }^{22}$

Wird in den vorliegenden Daten eine dialektale Ausprägung charakterisiert, die nur bedingt den vorhandenen Kategorien $\mathrm{zu}$ entsprechen scheint, so kommt beinahe ausnahmslos das Konzept der Mischung oder des Einschlags ins Spiel.

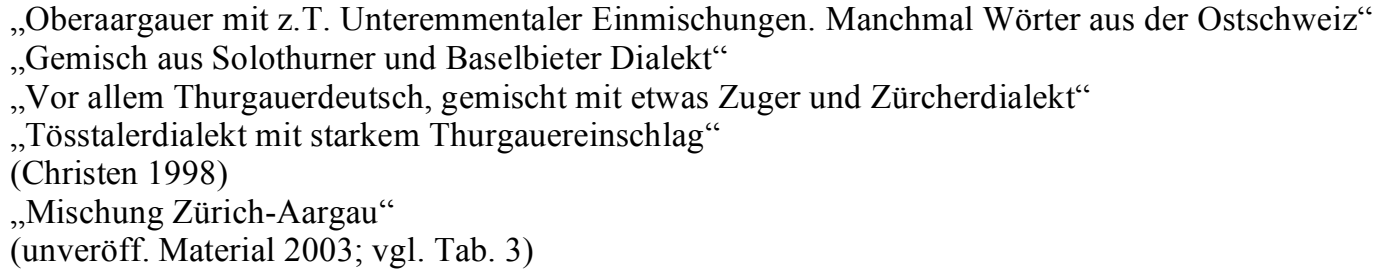

Dialekte werden als unterscheidbare Materialien konzeptualisiert, die sich so mischen lassen, dass die Materialien nicht zu einem neuen Stoff mit eigener Charakteristik verschmelzen, sondern die Ausgangsmaterialien sich in der resultierenden Mischung weiterhin identifizieren lassen. Dieselbe Vorstellung liegt Einschlag zugrunde. Die einzelnen Dialekte werden als Grössen konzeptualisiert, die eine materielle Kontur haben und gegeneinander abgrenzbar sind. Für die ethnolinguistische Konzeptualisierung der Deutschschweizer Sprachsituation ist entscheidend, dass Idiolekte, die als Mischungen qualifiziert werden, immer Dialekte bleiben, die in Beziehung gesetzt werden zu den idealen Kategorienvertretern, nämlich reinen Dialekten, und nicht etwa zur Standardsprache.

\section{Schluss}

Ethnolinguistischer Umgang mit Dialekten findet seinen Ausdruck unter anderem in Dialektbezeichnungen, die einen Reflex von alltagsweltlichen Dialekteinteilungen darstellen. Diese Dialekteinteilungen werden nicht nach einem bestimmten Merkmalset vorgenommen, sondern es handelt sich um natürliche, prototypisch organisierte Kategorien. In der Deutschschweiz werden in der Regel Kantonsmundarten als gestalthafte Grössen erfahren, nicht jedoch die Grössen vom Typ Deutschschweiz, von denen keine Vorstellung besteht. Die Kantone

21 Zum Zusammenhang von Dialektidealisierung und Dialektgebrauch der Eliten vgl. Haas (1992).

22 Unübersehbar ist, dass alltagsweltliche Konzeptualisierungen von Sprache als Ding und als Organismus ihre wissenschaftlichen Pendants haben. 
erweisen sich als primäre identitätsstiftende Kategorien, deren gesellschaftliche Relevanz einen Denkrahmen konstituiert, der (sprachliche) Differenzen alltagsweltlich zufrieden stellend ordnen kann.

Eine Reihe von Attribuierungen, mit denen Dialektbezeichnungen versehen werden, machen nochmals deutlich, dass Dialektkategorien prototypisch organisiert sind. Ausserdem zeigen sie auf, mit welchen Bildbereichen der Wirklichkeitsausschnitt Dialekt in seinen unterschiedlichen Erscheinungsformen alltagsweltlich erfasst wird und welche Dialektideologie verbreitet ist. In diesem Zusammenhang ist darauf hinzuweisen, dass das Attribut tief, das in Bezug auf deutschländische oder österreichische Dialekte sehr wohl verwendet wird (vgl. Beispiel <18>, sowie Tophinke \& Ziegler in diesem Band), in den hier untersuchten Daten nicht belegt ist.

Beispiel $<18>$

Der redet sicher immer im tiefsten dialekt. naja trotzdem, er hat was drauf.

www.hiphop.at/forum/international-rap/ohvo-5717.html - 65k

Breit mag im Deutschschweizer Kontext eine ähnliche Qualität bezeichnen wie tief in den anderen deutschsprachigen Ländern und einen hohen Anteil an sprachlichen Grössen meinen, die einen Dialekt in der alltagsweltlichen Wahrnehmung zu einem spezifischen Dialekt machen. Die vertikale Dimension, die mit tief, tiefst ins Spiel kommt und - vor einem anderen soziolinguistischen Hintergrund - der Wahrnehmung eines sozial bewerteten Hoch-Tief-Gefälles mit der Standardsprache als positivem Referenzpunkt Rechnung trägt, ist nicht verträglich mit der Deutschschweizer Dialektkonzeptualisierung, bei der die Vorstellung eines horizontalen Nebeneinanders von Dialekten dominiert, die jeweils zwar einem Dialektideal mehr oder weniger entsprechen können, aber ohne Frage immer als Dialekt aufgefasst werden.

\section{Literatur}

Assmann, Jan. 1992. Das kulturelle Gedächtnis. Schrift, Erinnerung und politische Identität in frühen Hochkulturen. München.

Auer, Peter. 2004. Sprache, Grenze, Raum. In: Zeitschrift für Sprachwissenschaft 23, 149-179.

Berthele, Raphael. 2006. Wie sieht das Berndeutsche so ungefähr aus? Über den Nutzen von Visualisierungen für die kognitive Laienlinguistik. In: Hubert Klausmann (Hg.). Raumstrukturen im Alemannischen. Graz-Feldkirch. 163-175.

Chambers, J. K. \& Peter Trudgill. 1980. Dialectology. Cambridge.

Christen, Helen. 1998. Dialekt im Alltag. Eine empirische Untersuchung zur lokalen Komponente heutiger schweizerdeutscher Varietäten. Tübingen.

Furrer, Norbert. 2002. Die vierzigsprachige Schweiz. Zürich.

Haas, Walter. 1992. Reine Mundart. In: Harald Burger u. a. (Hg.). Verborum amor. Studien zur Geschichte und Kunst der deutschen Sprache. Berlin. 578-610.

Lakoff, George. 1987. Women, Fire, and Dangerous Things. What Categories Reveal about Mind. Chicago, London.

Löffler, Heinrich. 2003. Dialektologie. Tübingen.

Muster, Hans Peter \& Beatrice Bürkli Flaig. 2001. Baselbieter Wörterbuch. Basel. 
Niebaum, Hermann \& Jürgen Macha. 2006. Einführung in die Dialektologie des Deutschen. 2. Aufl. Tübingen.

Niedzielski, Nancy A. \& Dennis R. Preston. 2003). Folk linguistics. Berlin, New York.

Paul, Hermann. 2002. Deutsches Wörterbuch. 9. Aufl. Tübingen.

Ris, Roland. 1992. Innerethik der deutschen Schweiz. In: P. Hugger (Hg.). Handbuch der schweizerischen Volkskultur. 3 Bde. Zürich. 749-766.

Ris, Roland. 1993. Schweizer Kantonsmundarten. In: Konturen. Magazin für Sprache, Literatur und Landschaft 3, 38.

Rosch, Eleanor. 1978. Principles of Categorization. In: Eleanor Rosch u. a. (Hg.). Cognition and Categorization. Hillsdale N.J.

Rosch, Eleanor \& C. Mervis \& W. Gray \& D. Johnson \& P. Boyes-Braem. 1976. Basic Objects in Natural Categories. In: Cognitive Psychology 8, 382-439.

Saeed, John I. 2003. Semantics. 2. Aufl. Oxford.

Schweizerisches Idiotikon. Wörterbuch der schweizerdeutschen Sprache. Frauenfeld. $1881 \mathrm{ff}$.

Stalder, Franz Joseph. 1819. Die Landessprachen der Schweiz oder Schweizerische Dialektologie mit kritischen Sprachbemerkungen beleuchtet. Nebst der Gleichnißrede von dem verlorenen Sohne in allen Schweizermundarten. Aarau.

Weber, Albert. 1987. Zürichdeutsche Grammatik. 2. Aufl. Zürich.

Weinrich, Harald. 2005. Textgrammatik der deutschen Sprache. 3. Aufl. Hildesheim.

Wiesinger, Peter. 1983. Die Einteilung der deutschen Dialekte. In: Werner Besch u. a. (Hg.). Dialektologie. Ein Handbuch zur deutschen und allgemeinen Dialektforschung. 2. Halbband. Berlin. 807-900.

Wrede, Ferdinand. 1956. Einführung zum Deutschen Sprachatlas. Zweiundzwanzigste und dreiundzwanzigste Lieferung. Marburg.

Zehetner, Ludwig. 1985. Das barische Dialektbuch. München.

Prof. Dr. Helen Christen

Universität Freiburg i. Üe.

Departement für Germanistik

Av. de l'Europe 20

CH-1700 Freiburg

helen.christen@unifr.ch 